\title{
Severe odontogenic infections: Epidemiological, microbiological and therapeutic factors
}

\author{
R. Sánchez ${ }^{1}$, E. Mirada ${ }^{1}$, J. Arias ${ }^{2}$, J.R. Paño ${ }^{3}$, M. Burgueño ${ }^{4}$
}

\author{
${ }^{1}$ Resident in training. Department of Oral and Maxillofacial Surgery. La Paz University Hospital. Madrid \\ ${ }^{2}$ Staff physician. Department of Oral and Maxillofacial Surgery. La Paz University Hospital. Madrid \\ ${ }^{3}$ Staff physician. Unit of Infectious Diseases and Clinical Microbiology. Department of Internal Medicine. La Paz University \\ Hospital. Madrid \\ ${ }^{4}$ Head of Department. Department of Oral and Maxillofacial Surgery. La Paz University Hospital. Madrid (Spain)
}

Correspondence:

Servicio de Cirugía Oral y Maxilofacial

Hospital Universitario La Paz

Paseo de la Castellana 261

28046 - Madrid (Spain)

ro_sb@hotmail.com

\author{
Sánchez R, Mirada E, Arias J, Paño JR, Burgueño M. Severe odontogenic \\ infections: Epidemiological, microbiological and therapeutic factors. Med \\ Oral Patol Oral Cir Bucal. 2011 Aug 1;16 (5):e670-6. \\ http://www.medicinaoral.com/medoralfree01/v16i5/medoralv16i5p670.pdf

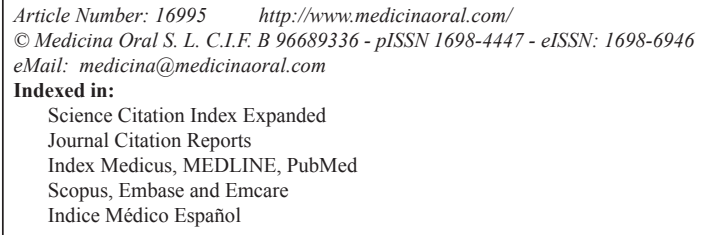

Received: 18/03/2010

Accepted: 21/04/2010

\begin{abstract}
Objectives: A retrospective study is made of the odontogenic infections treated in La Paz University Hospital (Madrid, Spain) during 2007 and 2008, with an epidemiological and microbiological analysis of a large group of patients.

Study Design: A retrospective descriptive study was made, involving the consecutive inclusion of all patients with odontogenic infection requiring admission to our hospital in the period between January 2007 and December 2008.

Results: A total of 151 patients were included, with a mean age of 40.3 years and a balanced gender distribution. The most frequently affected teeth were those located in the posterior mandibular segments, caries being the main underlying cause. Most isolates comprised mixed flora, particularly viridans streptococci, different species of Prevotella, Micromonas micros, and different species of Actinomyces. Susceptibility analysis of the microbial isolates showed a high percentage resistance to clindamycin ( $42.8 \%$ of all isolates), particularly among viridans streptococci.

Conclusions: The use of antibiotics in head and neck infections requires updated protocols based not only on the existing scientific evidence but also on the epidemiological reality of each center. On the other hand, identification is required of the surgical factors determining infection and how they influence morbidity associated with this type of pathology.
\end{abstract}

Key words: Odontogenic infection, odontogenic abscess, oral abscess, oral flora, oral pathogens, antibiotics. 


\section{Introduction}

Odontogenic infections are the main cause of consultation in dental practice, and one of the main causes of consultation in primary care. Such infections affect individuals of all ages, and account for approximately $10 \%$ of all antibiotic prescriptions in Spain (1). In turn, severe dental infections are responsible for many emergencies attended by oral and maxillofacial surgeons in the hospital setting.

Despite the epidemiological relevance of these infections, few studies in the literature have examined their epidemiological and/or microbiological characteristics, and there is little scientific evidence supporting standardized protocols for the treatment of such pathology. The present study offers a retrospective analysis of the odontogenic infections treated in La Paz University Hospital (Madrid, Spain) during the years 2007 and 2008, conducting an epidemiological and microbiological analysis of a large group of patients with a view to defining adequate and up to date management protocols.

\section{Material and Methods}

A retrospective descriptive study was made, involving the consecutive inclusion of all patients with odontogenic infection requiring admission to our hospital in the period between January 2007 and December 2008. The data were obtained from the patient case histories. The study sample consisted of 151 patients that were included after confirming the following inclusion criteria: 1) the presence of severe odontogenic infections; and 2) the need for hospital admission. The usual criteria for hospital admission in this type of pathology include: trismus (defined as an oral aperture of under $40 \mathrm{~mm}$ ), dysphagia, odynophagia, dyspnea, high fever (over $38^{\circ} \mathrm{C}$ ), worsened general condition or the need for hospital control of concomitant systemic diseases. One or more of these admission criteria were required for inclusion in the study.

The basic diagnostic protocol included an anamnesis, exploration of the oral cavity, and the use of imaging techniques. Regarding the imaging techniques, and in addition to a panoramic X-ray study, periapical or occlusal X-rays were obtained in some cases to complete the diagnosis. Computed tomography with contrast injection was used when collections or abscesses were suspected.

Emergency laboratory tests were also requested (complete blood count, coagulation tests and basic biochemistry).

The management protocol was based on intravenous antibiotic treatment, with or without surgery. The first empirical treatment option used in our Department during the study period consisted of amoxicillin - clavulanate at standard doses ( $1 \mathrm{~g}$ every 8 hours via the intravenous route). Our second empirical treatment option consisted of the combination of clindamycin $600 \mathrm{mg} / 8$ hours and gentamycin $240 \mathrm{mg} / 24$ hours, and was used in cases of beta-lactam allergy and in subjects receiving outpatient treatment with amoxicillin - clavulanate for prolonged periods of time (over 7 days), in which the clinical course showed scant improvement.

Patients receiving in-hospital treatment with amoxicillin - clavulanate and who failed to improve within 48 hours were switched to clindamycin plus gentamycin, or alternatively the latter antibiotic combination was added to amoxicillin - clavulanate, at the same described doses.

Medical management was completed with intravenous corticosteroids at a dose of $1 \mathrm{mg} / \mathrm{kg} / 8$ hours, followed by a decreasing dosing regimen in those patients at risk of airway problems or dysphagia. Intravenous analgesics and antiinflammatory drugs were used, associated to gastric protection with a proton pump inhibitor.

Surgery in turn was based on drainage of the underlying collection or abscess under local or general anesthesia. The causal tooth was removed in those cases where restorative dental treatment was ruled out. In patients subjected to the drainage of cervicofacial abscesses, a drain was placed in the anatomical space for several days. Samples for microbiological study were obtained in the case of purulent contents.

The demographic data collected were patient age and gender, and parameters relating to the medical history and known allergies. Data on the outpatient course of the process were collected, along with the patient signs and symptoms at the time of admission, the tooth or teeth involved, and the underlying causal mechanism. The causal teeth were divided into 6 groups: upper anterior teeth, upper posterior teeth, upper third molars, lower anterior teeth, lower posterior teeth and lower third molars.

Data in turn were collected on the duration of hospital stay, and the medical and surgical treatments provided. The microbiological variables included identification of the isolates and their drug sensitivity profile (antibiogram). The complications observed during hospital stay were also documented. Following hospital discharge, we collected information on those patients requiring readmission due to a non-satisfactory clinical course as evidenced on occasion of the ambulatory controls (Table 1).

A Microsoft Excel spreadsheet and the SPSS version 12.0 statistical package were used for statistical analysis. A descriptive study was made of all the variables included in the study protocol. Likewise, statistical analyses were carried out using the Mann-Whitney U-test, the Kruskal-Wallis test, and nonparametric tests. 
Table 1. Data collection.

\begin{tabular}{|c|c|}
\hline \multicolumn{2}{|r|}{ DATA COLLECTION } \\
\hline Anamnesis & $\begin{array}{l}\text { Age, gender } \\
\text { Personal history } \\
\text { Drug allergies }\end{array}$ \\
\hline Present illness & $\begin{array}{l}\text { Duration } \\
\text { Ambulatory antibiotic treatment and duration }\end{array}$ \\
\hline Physical examination & $\begin{array}{l}\text { Signs and symptoms: trismus, dyspnea, dysphagia, odynophagia } \\
\text { Causal tooth or teeth } \\
\text { Underlying dental pathology }\end{array}$ \\
\hline Complementary tests & $\begin{array}{l}\text { Panoramic X-rays } \\
\text { Computed axial tomography } \\
\text { Periapical, occlusal X-rays }\end{array}$ \\
\hline Hospital admission & $\begin{array}{l}\text { Admission date and duration of stay } \\
\text { Antibiotic treatment administered and duration } \\
\text { Corticosteroids }\end{array}$ \\
\hline Surgical procedure & $\begin{array}{l}\text { Date } \\
\text { Procedure } \\
\text { Local or general anesthesia } \\
\text { Drain placement }\end{array}$ \\
\hline Microbiology & $\begin{array}{l}\text { Aerobic, anaerobic or mixed flora } \\
\text { Genus and species } \\
\text { Antibiotic resistances }\end{array}$ \\
\hline Discharge & $\begin{array}{l}\text { Discharge date } \\
\text { Ambulatory antibiotic treatment and duration }\end{array}$ \\
\hline Complications & $\begin{array}{l}\text { Complications } \\
\text { Readmission } \\
\text { Reintervention }\end{array}$ \\
\hline
\end{tabular}

\section{Results}

During the study period, a total of 151 patients with complicated odontogenic infections were admitted: 81 males and 70 females, with a mean age of 40.39 years. Nineteen patients had medical antecedents affecting the integrity of the immune system. These included type 1 or 2 diabetes in 12 subjects $(7.95 \%$ of the overall study series). Among the 17 patients with known drug allergies, 9 were allergic to penicillins or beta-lactams. Most of the patients $(81.8 \%)$ presented a clinical course prior to hospital admission of less than 7 days, while $18.2 \%$ had presented symptoms for more than 7 days. A total of 104 patients received ambulatory antibiotic treatment via the oral route before admission to hospital (representing $68.9 \%$ of the global sample). In this subgroup, $77.9 \%$ of the patients received amoxicillin - clavulanate, either alone or in combination with another antibiotic.

Regarding the underlying pathogenic mechanisms, the most frequent triggering cause was dental caries (51 patients, representing $33.8 \%$ of all identified cases), followed by post-extraction infectious processes (18 cases) and pericoronitis (9 cases) (Fig. 1).

In relation to the teeth implicated in the infection, those located in the lower posterior segments (premolars and molars) were involved in $61.5 \%$ of the cases (85 patients). The second most frequent causal teeth were the lower third molars $(26.6 \%, 37$ cases $)$.
Regarding clinical presentation at the time of admission, 53 patients (35.1\% of the total) showed trismus - this being the most common clinical sign. In turn, 35 patients $(23.2 \%)$ suffered trismus associated to dysphagia.

During hospital admission, the most frequent antibiotic treatment was the combination clindamycin - gentamycin (50 patients, 33.1\%). This was followed in order of frequency by the combination amoxicillin - clavulanate (39 patients, 25.8\%). The association of amoxicillin clavulanate and clindamycin - gentamycin was the third most frequent treatment (34 patients, 22.5\%). In the rest of the patients we used other antibiotic combinations, including metronidazole, levofloxacin, piperacillin tazobactam or penicillin (Fig. 2).

Of the total study sample, 131 patients $(86.8 \%)$ underwent surgery in the first hours after admission, and following the pertinent complementary tests and confirmation of the need for drainage or extraction. In 39 cases $(29.7 \%)$ surgery was performed under general anesthesia, while local anesthesia was used in 92 cases (70.2\%). A group of 20 patients (13.2\%) received only conservative treatment with antibiotics.

As to the type of surgery involved, extraction of the causal tooth or teeth was carried out in 81 cases $(61.8 \%)$. In 30 cases $(22.9 \%)$, both extraction and collection or abscess drainage were carried out, while drainage alone was carried out in 18 cases $(13.7 \%)$.

At discharge, 75 patients (49.7\%) were prescribed am- 


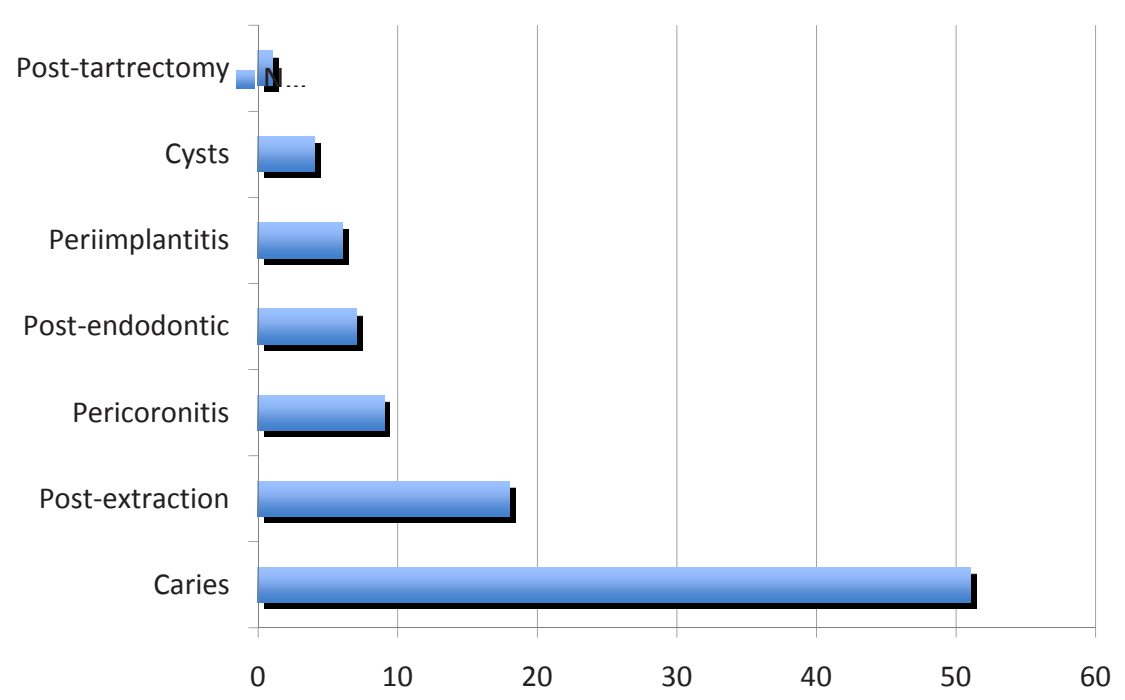

Fig. 1. Patient distribution according to identified cause of odontogenic infection.

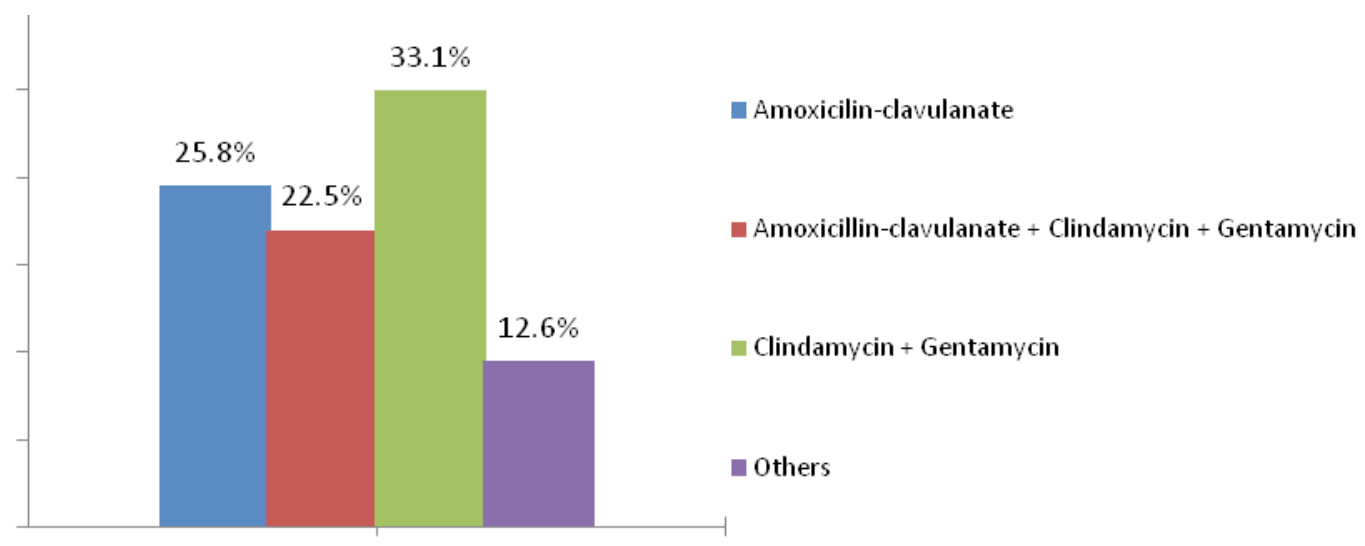

Antibiotic treatment

Fig. 2. In-hospital antibiotic treatment provided.

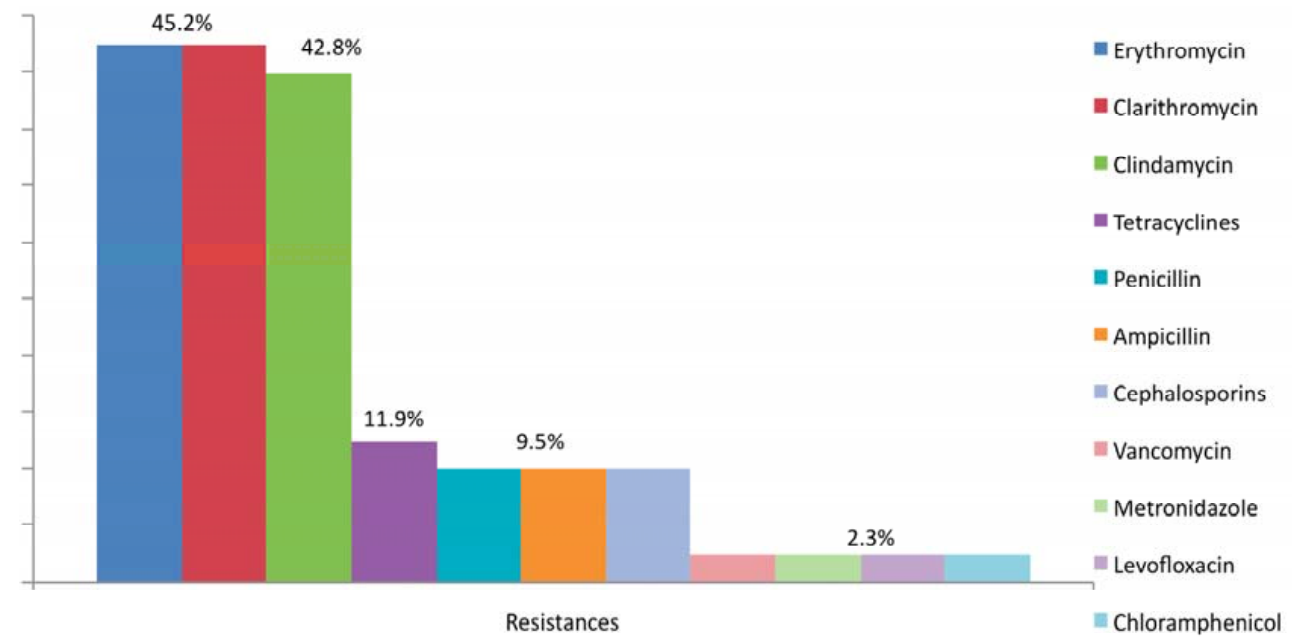

Fig. 3. Distribution of samples with associated resistances. 
Table 2. Distribution of isolated microorganisms.

\begin{tabular}{|l|c|c|}
\hline \multicolumn{1}{|c|}{ AEROBES } & N (isolates) & \% total isolates (N=126) \\
\hline Streptococcus & 34 & $26.9 \%$ \\
$\bullet \quad$ Viridans group & 32 & $25.3 \%$ \\
$1.5 \%$
\end{tabular}

\begin{tabular}{|l|c|c|}
\hline \multicolumn{1}{|c|}{ ANAEROBES } & N (isolates) & \% total isolates (N=126) \\
\hline Prevotella & 33 & $26.1 \%$ \\
\hline Micromonas micros & 13 & $10.3 \%$ \\
\hline Actinomyces & 11 & $8.7 \%$ \\
\hline Eikenella corrodens & 4 & $3.1 \%$ \\
\hline Lactobacillus & 4 & $3.1 \%$ \\
\hline Fusobacterium necrophorum & 3 & $2.3 \%$ \\
\hline Finegoldia magna & 3 & $2.3 \%$ \\
\hline Propionebacterium acnes & 2 & $1.5 \%$ \\
\hline Peptostreptococcus anaerobis & 2 & $1.5 \%$ \\
\hline Bacteroides & 2 & $1.5 \%$ \\
\hline Porphyromona asaccharolytica & 1 & $0.7 \%$ \\
\hline Bifidobacterium & 1 & $0.7 \%$ \\
\hline Genella morbillorum & 1 & $0.7 \%$ \\
\hline TOTAL & $\mathbf{8 0}$ & $\mathbf{6 3 . 4 \%}$ \\
\hline & $\mathbf{N}$ (isolates) & $\mathbf{\%}$ total isolates (N=126) \\
\hline Yeasts & 3 & $2.3 \%$ \\
\hline Candida albicans & 1 & $0.7 \%$ \\
\hline TOTAL & $\mathbf{4}$ & $\mathbf{3 . 1 \%}$ \\
\hline
\end{tabular}

bulatory treatment with clindamycin, while 70 patients (46.4\%) received amoxicillin - clavulanate.

The mean stay was 4.24 days (range 1-31 days). In the subgroup of patients with type 1 or 2 diabetes, the mean stay was 9.2 days (with over 15 days in three cases). Four patients required repeat surgery, and in 5 cases patient readmission proved necessary. Regarding other complications, we documented a single case of mandibular osteomyelitis.

There was no statistically significant correlation between the antibiotic treatment used and the duration of hospital stay. Likewise, no significant correlation was found between the patients subjected or not subjected to surgery and the duration of hospital stay.

In the subgroup of patients readmitted to hospital (5 cases), the mean age was 36 years, with a mean duration or first hospital stay of 11.4 days. In all cases the reason for readmission was a non-satisfactory clinical course after discharge; reappearance of the abscess was ob- served in one case (requiring repeat surgery), while the rest of the subjects received antibiotic treatment. The microbiological study of the cases requiring readmission revealed the presence of Prevotella intermedia in one case, and of Streptococcus intermedius resistant to clindamycin, clarithromycin and erythromycin in another.

A total of 48 samples for microbiological study were obtained. Of these, 6 proved insufficient, showed no growth, or were seeded in inappropriate media, and were therefore discarded. Grampositive bacilli were isolated in 7 cases, gramnegative bacilli in 43 cases, grampositive cocci in 59 cases, and gramnegative cocci in two cases. In addition, four microorganisms presenting hyphae were isolated. Mixed flora was isolated in 20 samples $(47.62 \%)$, while aerobes alone were isolated in 14 cases $(33.33 \%)$, and anaerobes in 8 samples $(19.05 \%)$. The most frequently isolated organisms were different species of Prevotella (33 isolates) and different species 
of viridans streptococci (32 isolates). High prevalence was also recorded for Micromonas micros (13 isolates), different species of Actinomyces (11 isolates), and different species of Staphylococcus (6 isolates) (Table 2).

Regarding the detected antibiotic resistances, $45.2 \%$ of the samples contained one or more germs resistant to erythromycin, while the same percentage showed resistance to clarithromycin. A total of $42.8 \%$ of the samples contained one or more microorganisms resistant to clindamycin. In turn, resistance to tetracyclines was detected in $11.9 \%$ of the samples, while resistance to penicillin, ampicillin and cephalosporins was observed in $9.5 \%$ of the samples (Fig. 3).

The analysis of the microorganisms responsible for the high proportion of clindamycin resistance showed viridans streptococci to be implicated in $63 \%$ of all cases.

\section{Discussion}

Odontogenic infections are among the conditions most commonly treated by oral and maxillofacial surgeons. These infections affect a large portion of the population, and can give rise to serious complications if not treated quickly and adequately. Thus, the importance of infections of dental origin is their high incidence and morbidity (1-3).

In our series the mean patient age was 40.3 years, which is somewhat older than in other studies (3-6). In contrast to other series in which a male predominance has been observed $(3,5)$, the two genders were equally represented in our study.

Considering disease antecedents implying an altered immune system, our subgroup of patients with type 1 or 2 diabetes mellitus represented almost $8 \%$ of the total series, while in the general population the prevalence of type 2 diabetes is about $10-15 \%$ (6). In this subgroup of patients, the mean duration of hospital stay was 9.2 days, which was longer than for the global sample (4.24 days). As in other studies, diabetic patients were more vulnerable and were associated to longer mean hospital stays (7).

In our study the most frequent cause of infection was dental caries (33.8\%), followed by post-extraction infectious processes and pericoronitis. The most frequently implicated teeth were those located in the lower posterior segments (61.5), followed by the lower molars (26.6\%). In contrast, other studies have found the lower molars to be the most frequent causal teeth $(8-10)$. This predominant location in posterior sectors could be due to the increased technical difficulty of restorative treatments in this zone, and to less thorough oral hygiene in the posterior regions of the oral cavity.

As regards clinical presentation of the infection, 35.1\% of the patients showed trismus alone, while $23.2 \%$ suffered trismus associated to dysphagia - in coincidence with the observations of other authors $(4,7)$. Despite the clinical importance of these signs and symptoms, they were not present in a large proportion of patients at the time of admission to hospital. In any case, long-evolving infections, a torpid clinical course, or the need to control other concomitant diseases in many cases make admission necessary even in the absence of such clear manifestations.

During hospital admission, the patients were treated with intravenous antibiotics, and the majority also received surgery $(86.8 \%)$ - mostly under local anesthesia $(70.2 \%$ versus general anesthesia in $29.7 \%)$. Surgery usually consisted of extraction of the causal tooth (61.8\% of the cases), with the association of collection or abscess drainage in $22.9 \%$ of the cases. According to the literature, surgical drainage is needed in $10-83 \%$ of all cases $(4,5,10)$. The indications of drainage are based on the clinical or radiological evidence of a collection or abscess. Abscess spread to more than one anatomical space, the risk of airway involvement, and the patient anesthesia risk are determinant factors in deciding to use local or general anesthesia.

In our study, the most frequently isolated organisms were viridans streptococci $25.3 \%$ of all isolated germs). The identified anaerobes were different species of Prevotella (26.1\%), Micromonas micros (10.3\%), and different species of Actinomyces (8.7\%). The normal oropharyngeal flora was thus seen to predominate, with a high frequency of polymicrobial infections caused by aerobes and anaerobes, in almost one-half of all the samples $(47.62 \%)$. These results agree with those reported by other authors (1, 11-17).

The treatment protocol used in our center includes amoxicillin - clavulanate as first choice antibiotic therapy in disorders of this kind. However, in the present study the most widely used drug combination was clindamycin - gentamycin $(33.1 \%$ versus $25.8 \%$ for amoxicillin - clavulanate, and $22.5 \%$ for both combinations together). This can be explained by the high percentage of patients treated with amoxicillin - clavulanate on an ambulatory basis during the period prior to hospital admission (40.4\%), either alone or combined with other antibiotics.

In these patients with clinical worsening despite treatment with an antibiotic for a prolonged period of time (over 7 days), our protocol contemplates a change in therapy - introducing clindamycin associated to gentamycin (i.e., the second drug treatment choice in our center). This protocol coincides with the drug treatment regimens of other studies in our country, which corroborate the suitability of these two antibiotics in the management of odontogenic infections $(1,17,18)$.

However, no statistically significant correlation was observed between the mean hospital stay and the type of antibiotic administered, or treatment with or without surgery. Further studies are needed to define the epi- 
demiological, clinical and therapeutic factors with the greatest impact upon patient morbidity-mortality.

The administration of penicillins (penicillin, ampicillin and amoxicillin) is considered to be useful for the empirical management of odontogenic infections, as described by other studies published in Spain and particularly in other countries $(1,4,12-14,17,19)$. The advantages of such treatment are based on its appropriate spectrum, few side effects and low cost. However, some studies have alerted to an increase in the incidence of beta-lactam resistant organisms, particularly betalactamase producing anaerobes and viridans streptococci, which are frequently found in odontogenic infections $(1,7,15,16)$. In this study, penicillin and ampicillin resistance was found in $9.5 \%$ of the samples.

Many studies describe clindamycin as the treatment of choice among patients with allergy to beta-lactams, or as a second drug option following treatment failure in patients administered penicillins $(1,4,12)$. The drug offers good activity against anaerobes (with the important exception of Eikenella corrodens), Streptococcus and methicillin-sensitive Staphylococcus. However, a number of studies have reported an increase in clindamycin resistance among anaerobes and viridans streptococci $(1,17,20)$. According to Kuriyama et al. (10), the clindamycin sensitivity rate for this latter group of bacteria is $54 \%$, while the drug shows good activity against anaerobes - particularly against certain species of Prevotella $(12,20)$.

In our study, resistance to clindamycin was recorded in $42.8 \%$ of the samples analyzed - viridans streptococci in particular being responsible for $63 \%$ of all such cases.

In conclusion, the use of antibiotics in head and neck infections requires updated protocols based on the existing scientific evidence relating to pathogen profile and resistances. On the other hand, identification is required of the surgical factors determining infection and how they influence morbidity associated with this type of pathology.

\section{References}

References with links to Crossref - DOI

1. Bascones Martínez A, Aguirre Urízar JM, Bermejo Fenoll A, Blanco Carrión A, Gay-Escoda C, González-Moles MA, et al. Consensus statement on antimicrobial treatment of odontogenic bacterial infections. Med Oral Patol Oral Cir Bucal. 2004;9:369-76; 363-9.

2. Carter LM, Layton S. Cervicofacial infection of dental origin presenting to maxillofacial surgery units in the United Kingdom: a national audit. Br Dent J. 2009;206:73-8.

3. Huang TT, Tseng FY, Liu TC, Hsu CJ, Chen YS. Deep neck infection in diabetic patients: comparison of clinical picture and outcomes with nondiabetic patients. Otolaryngol Head Neck Surg. 2005;132:943-7.

4. Sato FR, Hajala FA, Freire Filho FW, Moreira RW, de Moraes M. Eight-year retrospective study of odontogenic origin infections in a postgraduation program on oral and maxillofacial surgery. J Oral Maxillofac Surg. 2009;67:1092-7.

5. Wang J, Ahani A, Pogrel MA. A five-year retrospective study of odontogenic maxillofacial infections in a large urban public hospital. Int J Oral Maxillofac Surg. 2005;34:646-9.

6. Valdés S, Rojo-Martínez G, Soriguer F. Evolution of prevalence of type 2 diabetes in adult Spanish population. Med Clin (Barc). 2007 Sep 15;129(9):352-5. Review. Spanish. Erratum in: Med Clin (Barc). 2007;129:599.

7. Flynn TR, Shanti RM, Levi MH, Adamo AK, Kraut RA, Trieger N. Severe odontogenic infections, part 1: prospective report. J Oral Maxillofac Surg. 2006 ;64:1093-103.

8. Haug RH, Hoffman MJ, Indresano AT. An epidemiologic and anatomic survey of odontogenic infections. J Oral Maxillofac Surg. 1991;49:976-80.

9. Osborn TM, Assael LA, Bell RB. Deep space neck infection: principles of surgical management. Oral Maxillofac Surg Clin North Am. 2008;20:353-65.

10. Kuriyama T, Karasawa T, Nakagawa K, Saiki Y, Yamamoto E, Nakamura S. Bacteriologic features and antimicrobial susceptibility in isolates from orofacial odontogenic infections. Oral Surg Oral Med Oral Pathol Oral Radiol Endod. 2000;90:600-8.

11. Flynn TR, Shanti RM, Hayes C. Severe odontogenic infections, part 2: prospective outcomes study. J Oral Maxillofac Surg. 2006;64:1104-13.

12. Warnke PH, Becker ST, Springer IN, Haerle F, Ullmann U, Russo PA, et al. Penicillin compared with other advanced broad spectrum antibiotics regarding antibacterial activity against oral pathogens isolated from odontogenic abscesses. J Craniomaxillofac Surg. 2008;36:462-7.

13. Rega AJ, Aziz SR, Ziccardi VB. Microbiology and antibiotic sensitivities of head and neck space infections of odontogenic origin. J Oral Maxillofac Surg. 2006;64:1377-80.

14. Sixou JL, Magaud C, Jolivet-Gougeon A, Cormier M, BonnaureMallet M. Microbiology of mandibular third molar pericoronitis: incidence of beta-lactamase-producing bacteria. Oral Surg Oral Med Oral Pathol Oral Radiol Endod. 2003;95:655-9.

15. Stefanopoulos PK, Kolokotronis AE. The clinical significance of anaerobic bacteria in acute orofacial odontogenic infections. Oral Surg Oral Med Oral Pathol Oral Radiol Endod. 2004;98:398-408.

16. Koeth LM, Good CE, Appelbaum PC, Goldstein EJ, Rodloff AC, Claros M, et al. Surveillance of susceptibility patterns in 1297 European and US anaerobic and capnophilic isolates to co-amoxiclav and five other antimicrobial agents. J Antimicrob Chemother. 2004;53:1039-44.

17. Brescó-Salinas M, Costa-Riu N, Berini-Aytés L, Gay-Escoda C. Antibiotic susceptibility of the bacteria causing odontogenic infections. Med Oral Patol Oral Cir Bucal. 2006;11:E70-5.

18. Isla A, Canut A, Rodríguez-Gascón A, Labora A, ArdanzaTrevijano B, Solinís MA, et al. Pharmacokinetic/pharmacodynamic analysis of antibiotic therapy in dentistry and stomatology. Enferm Infecc Microbiol Clin. 2005;23:116-21.

19. Poveda Roda R, Bagan JV, Sanchis Bielsa JM, Carbonell Pastor E. Antibiotic use in dental practice. A review. Med Oral Patol Oral Cir Bucal. 2007;12:E186-92.

20. Rodríguez-Avial I, Rodríguez-Avial C, Culebras E, Benítez A, Picazo JJ. Distribution of $\operatorname{mef}(\mathrm{A})$ and erm(B) genes in macrolideresistant blood isolates of viridans group streptococci. J Antimicrob Chemother. 2001;47:727-8. 